Piekarska Ewa, Pawlicki Mateusz, Lopuszyńska Anna, Kozioł Magdalena, Krasa Aleksandra, Piecewicz-Szczęsna Halina. Caffeine in Pain Perception and Management. Journal of Education, Health and Sport. 2021;11(8):328-333. eISSN 2391-8306. DOI http://dx.doi.org/10.12775/JEHS.2021.11.08.036

https://apcz.umk.pl/czasopisma/index.php/JEHS/article/view/JEHS.2021.11.08.036

https://zenodo.org/record/5274050

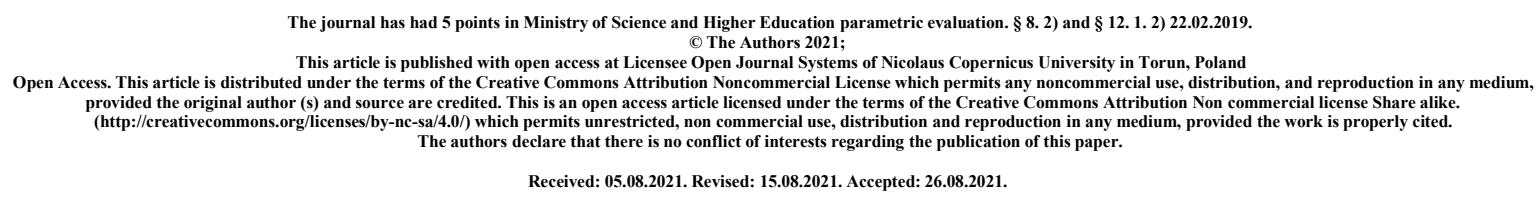

\title{
Caffeine in Pain Perception and Management
}

\section{Ewa Piekarska $^{1}$, Mateusz Pawlicki ${ }^{1}$, Anna Lopuszyńska ${ }^{1}$, Magdalena Koziol ${ }^{1}$, Aleksandra Krasa ${ }^{1}$, Halina Piecewicz-Szczęsna ${ }^{2}$}

\footnotetext{
${ }^{1}$ Student Scientific Association at Department of Epidemiology and Clinical Research Methodology Medical University of Lublin, ul. Radziwiłłowska 11, Lublin 20-080, Poland

${ }^{2}$ Department of Epidemiology and Clinical Research Methodology of the Medical University of Lublin, ul. Radziwiłłowska 11, Lublin 20-080, Poland
}

Corresponding author: Ewa Piekarska, piekarskaewaa@gmail.com

\section{ORCID ID:}

Ewa Piekarska https://orcid.org/0000-0002-4954-379X; piekarskaewaa@gmail.com Mateusz Pawlicki https//orcid.org/0000-0001-8318-6573, pawlak32@gmail.com Anna Łopuszyńska https://orcid.org/0000-0001-5133-4180, lopuszynskaania@gmail.com Magdalena Kozioł https://orcid.org/0000-0002-8671-5968, magdalena.koziol@icloud.com Aleksandra Krasa https://orcid.org/0000-0002-0733-202X, ola.AK62@gmail.com

\begin{abstract}
:
Introduction: Caffeine is considered to be one of the most frequently used pharmacologically active substances in the world. Caffeine is a psychoactive and central nervous system stimuli that has the ability to increase energy, decrease fatigue, promote alertness and wakefulness. Potential role of caffeine's nociceptive properties shows that it may play a relevant role in pain modulation. Although caffeine at dietary levels is not a sufficient analgesic in its own, it has been included in many over-the-counter and prescription drugs. This review addresses caffeine's analgesic properties in pain management.
\end{abstract}


Results: Caffeine shows relevant role in pain modulation through blocking adenosine receptors which are involved in nociception. Studies show that habitual dietary caffeine consumption results in decreased pain sensitivity. As an adjuvant to commonly used analgesics, caffeine provides an important increase in pain relief in various types of pain such as postoperative dental pain, headaches, or fibromyalgia.

Conclusions: In conclusion, caffeine is associated with decreased pain sensitivity. Addition of caffeine to analgesics has resulted in their enhanced analgesic efficacy in pain treatment.

Key words: caffeine; pain; analgesia

\section{Introduction:}

Caffeine is a naturally occurring substance found in seeds, fruit and leaves of plants such as tea, coffee beans and cocoa nuts. The most common dietary sources of caffeine include tea, coffee, soft and energy drinks or foods like chocolate [1]. Consumption of caffeine varies by a country and region. Each day approximately $80 \%$ of adults consume a caffeinated product [2]. Intake of $400 \mathrm{mg}$ caffeine per day (that's about four or five cups of coffee) has shown no serious adverse effects in healthy, non pregnant adults . Therefore the dose is considered harmless and does not raise safety concerns [3].

Caffeine is a psychoactive and central nervous system stimulant. Pure caffeine forms white, prismatic, odorless crystals, has a slightly bitter taste, and is slightly acidic [4]. It is completely absorbed in the intestinal tract [5]. Caffeine is frequently used by consumers for its ability to increase energy, decrease fatigue, promote alertness and wakefulness [6]. It also enhances physical performance, cognitive performance and short-term memory [7]. However, high quantity ( above $400 \mathrm{mg} /$ day) of caffeine may cause adverse effects such as: insomnia, restlessness, upset stomach, tremor or caffeine withdrawal syndrome e.g., headache, irritability, fatigue [8].

In addition to being a nervous system stimulant caffeine shows pain relieving properties. The nociceptive effects of caffeine are associated with its nonselective antagonism of the adenosine A1, A2A, A2B and A3 receptor [9]. Adenosine is an inhibitor of neuronal activity in the peripheral and central nervous systems. The structure of caffeine is similar to adenosine and therefore caffeine seems to express its direct effect via central blocking adenosine receptors that influence pain signaling or by blocking peripheral adenosine receptors on sensory afferents [10].

Although caffeine at dietary levels is not usually regarded as an analgesic in its own it has been included in many over-the-counter and prescription drugs [11,12].

Based on the idea that it enhances analgesic efficacy it became added as an adjuvant to popular drugs such as acetaminophen, ibuprofen, acetylsalicylic acid that are used to treat common painful conditions for example headache, postpartum pain, and postoperative pain. An adjuvant in this context is an agent that enhances the effects of a drug while having few if any direct effects when given by itself [9]. 
The exact adjuvant properties of caffeine are still not well understood. Possible mechanisms of action include:

- lowering gastric $\mathrm{pH}$ and increasing gastric blood flow which contribute to improved drug absorption

- $\quad$ reduced hepatic blood flow contributing to reduced metabolic drug clearance

- blocking nociceptive adenosine signaling

- inhibition of cyclooxygenase 2 (COX-2) activity

- mood and emotion state changes leading to change in perception of pain $[11,14]$.

Following given information on caffeine nociceptive and possible adjuvant properties a question arises: how does caffeine influence our pain perception and management?

\section{Review of available research:}

The observational study carried by the University of Alabama at Birmingham was to determine whether habitual consumption of caffeine was associated with experimental pain sensitivity. 62 participants monitored their daily caffeine consumption (e.gcoffee, tea, soda, energy drinks, and chocolate) for a period of seven days using a caffeine consumption diary. On the seventh day of caffeine consumption monitoring, participants completed pain sensitivity testing, using heat and pressure stimuli.

The study shows that each additional $100 \mathrm{mg}$ of daily caffeine consumed was associated with a $0.50 \mathrm{C}$ increase in heat pain threshold, a $0.20 \mathrm{C}$ increase in heat pain tolerance, and a $31.2 \mathrm{kPa}$ increase in pressure pain threshold at the trapezius. In conclusion increasing amounts of caffeine consumed habitually as part of a daily diet is associated with decreased pain sensitivity, represented in this study as higher pain thresholds and higher pain tolerance [16].

Following the prospect of caffeine's analgesic properties it may become relevant for people suffering from chronic or recurrent pain such as migraines or tension type headaches (TTH). Around $11 \%$ of adults worldwide suffer from migraine and at least $40 \%$ meet criteria for TTH. Baratloo et al. enrolled 61 patients diagnosed with migraine using International Headache Society criteria. Patients received $60 \mathrm{mg}$ caffeine citrate intravenously (i.v.). Pain scores were measured at baseline, $1 \mathrm{~h}$ and $2 \mathrm{~h}$ after receiving the dose using visual analog scale (VAS). The dose of $60 \mathrm{mg}$ caffeine citrate i.v. was well tolerated and safe. Patients with acute migraine headache showed significant pain relief ( after $1 \mathrm{~h}$ and $2 \mathrm{~h}$ ) [17].

Significant effect of caffeine in headache treatment was also presented in randomised, placebo-controlled, double-blind, counterbalanced-crossover study. Crystal F. Haskell-Ramsay et at. compared the effects of regular coffee, decaffeinated coffee, and placebo on measures of cognition and mood. Headache ratings were significantly lower in participants consuming regular coffee compared to decaffeinated and placebo [18].

While monotherapy with analgesic alone is proven to be efficient in pain treatment, there is good evidence that combining analgesics with caffeine can provide better pain relief [12].

Clinical study carried out by $\mathrm{T}$. Weiser et al. was conducted to assess efficiency of ibuprofen and caffeine combination in patients with post-surgical dental pain. 
Patients qualified for study underwent a third molar extraction procedure and received either one single dose of ibuprofen $400 \mathrm{mg} /$ caffeine $100 \mathrm{mg}$, ibuprofen $400 \mathrm{mg}$, caffeine $100 \mathrm{mg}$ or placebo. During trial pain severity was measured periodically by 4-point verbal rating scale (VRS) which was based on a written selection of options of 'no pain', 'slight pain', 'moderate pain', or 'severe pain' and the 0-10 numerical pain rating scale (NPRS) ranging from $0=$ 'no pain' to $10=$ 'worst possible pain'. The study demonstrated superior efficacy of the fixed dose combination (FDC) of $400 \mathrm{mg}$ ibuprofen and $100 \mathrm{mg}$ caffeine over either single compound and placebo in the dental impaction model. The FDC ibuprofen/caffeine provided approximately 30\% higher pain reduction/pain relief than ibuprofen alone on average over $8 \mathrm{~h}$. The analysis of various endpoints showed that the FDC provided faster pain reduction than ibuprofen alone. Median time to meaningful pain relief was 39 min earlier for patients taking the FDC, compared to those taking ibuprofen [20].

Very similar results have come up in Post Hoc Subgroup Analysis of a Randomised Controlled Trial conducted by Stefanie Förderreuther et al.. After dental surgery, patients received a single dose of ibuprofen $400 \mathrm{mg} /$ caffeine $100 \mathrm{mg}$, ibuprofen $400 \mathrm{mg}$, caffeine 100 mg or placebo. Results showed enhanced analgesic effect of ibuprofen plus caffeine versus ibuprofen, concluding that the efficacy is higher in patients with moderate and severe pain [21].

Postdural puncture headache is a common complication in patients who received spinal anesthesia. Recent study by Ashraf Ragab \& Khalid Noman Facharzt was evaluating the effect of caffeine sodium benzoate for postdural puncture headache prevention. Patients received $10 \mathrm{ml}$ normal saline or $10 \mathrm{ml}$ with $500 \mathrm{mg}$ caffeine sodium benzoate, intravenously, 60 min after administering spinal anesthesia. The patients' headaches were measured by using the visual analog scale (VAS). VAS were significantly lower in caffeine group than in saline group . There were also more incidents of moderate and severe headache in the group compared with the caffeine group, where Analgesic demand was significantly lower. In summary episodes of postdural puncture headache decreases in patients who received caffeine sodium benzoate [22].

Caffeine analgesic adjuvant properties were also addressed in opioid-using and non-using patients with fibromyalgia. Patients were grouped by opioid use and next split into groups based on caffeine consumption per day (no caffeine, low, moderate, high caffeine). Results of the study indicate caffeine adjuvant efficiency is higher in opioid users compared to non-users. Patients who were on opioid plus caffeine therapy reported less pain severity and depression, as well as higher physical function, compared to patients taking opioids but not consuming caffeine.

Non opioid users who consumed caffeine showed no differences in pain and symptoms compared to no caffeine grup. These data suggest that caffeine combined with opioid analgesics could provide therapeutic benefits in fibromyalgia treatment [23].

\section{Conclusions:}


Studies show clinically relevant superiority of caffeine plus analgesics over monotherapy with analgesic alone (in patients suffering from a variety of pain conditions). Although caffeine by itself isn't used as a standard pain treatment, it has shown positive effects on pain perception, as it decreases pain sensitivity. Physicians should keep in mind that the addition of caffeine in pain treatment may benefit certain patients, considering its ability to increase levels and reduce pain relief.

\section{References:}

1. Evans J, Richards JR, Battisti AS. Caffeine. [Updated 2020 Dec 2]. In: StatPearls [Internet]. Treasure Island (FL): StatPearls Publishing; 2021 Jan-. Available from: https://www.ncbi.nlm.nih.gov/books/NBK519490/

2. Ogawa N, Ueki H. Clinical importance of caffeine dependence and abuse. Psychiatry Clin Neurosci. 2007;61(3):263-268. doi: 10.1111/j.1440-1819.2007.01652.x.

3. Verster JC, Koenig J. Caffeine intake and its sources: A review of national representative studies. Crit Rev Food Sci Nutr. 2018;58(8):1250-1259. doi:10.1080/10408398.2016.1247252

4. PubChem, Caffeine (2015) Available at: http://pubchem.ncbi.nlm.nih.gov/summary/summary.cgi? $q=a l l \& c i d=2519 \#$. Accessed May 2021

5. Blanchard J, Sawers SJ. The absolute bioavailability of caffeine in man. Eur J Clin Pharmacol. 1983;24(1):93-98. doi: 10.1007/BF00613933.

6. McLellan TM, Caldwell JA, Lieberman HR. A review of caffeine's effects on cognitive, physical and occupational performance. Neurosci Biobehav Rev. 2016 Dec;71:294312. doi: 10.1016/j.neubiorev.2016.09.001. Epub 2016 Sep 6. PMID: 27612937.

7. Glade MJ. Caffeine-Not just a stimulant. Nutrition. 2010 Oct;26(10):932-8. doi: 10.1016/j.nut.2010.08.004. PMID: 20888549.

8. Cappelletti S, Piacentino D, Sani G, \& Aromatario M (2015). Caffeine: cognitive and physical performance enhancer or psychoactive drug? Curr Neuropharmacol, 13(1), 71-88. 10.2174/1570159X13666141210215655

9. Lipton, Richard B et al. "Caffeine in the management of patients with headache." The journal of headache and pain vol. 18,1 107. 24 Oct. 2017, doi:10.1186/s10194-017-0806-2

10. Baratloo A, Rouhipour A, Forouzanfar MM, Safari S, Amiri M, Negida A. The Role of Caffeine in Pain Management: A Brief Literature Review. Anesth Pain Med. 2016;6(3):e33193. Published 2016 Mar 26. doi:10.5812/aapm.33193

11. Derry CJ, Derry S, Moore RA. Caffeine as an analgesic adjuvant for acute pain in adults. Cochrane Database of Systematic Reviews 2014, Issue 12. Art. No.: CD009281. DOI: 10.1002/14651858.CD009281.pub3. Accessed 27 March 2021.

12. Derry S, Wiffen PJ, Moore RA. Single dose oral ibuprofen plus caffeine for acute postoperative pain in adults. Cochrane Database of Systematic Reviews 2015, Issue 7. Art. No.: CD011509. DOI: 10.1002/14651858.CD011509.pub2.

13. Baratloo A, Rouhipour A, Forouzanfar MM, Safari S, Amiri M, Negida A. The Role of Caffeine in Pain Management: A Brief Literature Review. Anesth Pain Med. 2016;6(3):e33193. Published 2016 Mar 26. doi:10.5812/aapm.33193 
14. Overstreet DS, Penn TM, Cable ST, Aroke EN, Goodin BR. Higher habitual dietary caffeine consumption is related to lower experimental pain sensitivity in a community-based sample. Psychopharmacology (Berl). 2018;235(11):3167-3176. doi:10.1007/s00213-0185016-3

15. Baratloo A, Negida A, El Ashal G. Intravenous Caffeine for the Treatment of Acute Migraine: A Pilot Study. J Caffeine Res. 2015;5(3):125-9. doi: 10.1089/jcr.2015.0004.

16. Haskell-Ramsay CF, Jackson PA, Forster JS, Dodd FL, Bowerbank SL, Kennedy DO. The Acute Effects of Caffeinated Black Coffee on Cognition and Mood in Healthy Young and Older Adults. Nutrients. 2018;10(10):1386. Published 2018 Sep 30. doi:10.3390/nu10101386

17. Weiser T, Richter E, Hegewisch A, Muse DD, Lange R. Efficacy and safety of a fixed-dose combination of ibuprofen and caffeine in the management of moderate to severe dental pain after third molar extraction. Eur J Pain. 2018;22(1):28-38. doi:10.1002/ejp.1068

18. Förderreuther S, Lampert A, Hitier S, Lange R, Weiser T. The Impact of Baseline Pain Intensity on the Analgesic Efficacy of Ibuprofen/Caffeine in Patients with Acute Postoperative Dental Pain: Post Hoc Subgroup Analysis of a Randomised Controlled Trial. Adv Ther. 2020;37(6):2976-2987. doi:10.1007/s 12325-020-01297-y

19. Ashraf Ragab \& Khalid Noman Facharzt (2014) Caffeine, Is it effective for prevention of postdural puncture headache in young adult patients?, Egyptian Journal of Anaesthesia, 30:2, 181-186, DOI: 10.1016/j.egja.2013.11.005

20. Scott JR, Hassett AL, Brummett CM, Harris RE, Clauw DJ, Harte SE. Caffeine as an opioid analgesic adjuvant in fibromyalgia. J Pain Res. 2017;10:1801-1809. Published 2017 Jul 28. doi:10.2147/JPR.S134421 